\title{
Appareil
}

$20 \mid 2018$

\section{Arts contemporains et patrimoine}

\section{Prise de site}

Jean-Louis Déotte

\section{(2) OpenEdition}

Journals

Édition électronique

URL : http://journals.openedition.org/appareil/2849

DOI : 10.4000/appareil.2849

ISSN : 2101-0714

Éditeur

MSH Paris Nord

Référence électronique

Jean-Louis Déotte, «Prise de site », Appareil [En ligne], 20 | 2018, mis en ligne le 25 octobre 2018, consulté le 30 juillet 2020. URL : http://journals.openedition.org/appareil/2849 ; DOI : https://doi.org/ 10.4000/appareil.2849

Ce document a été généré automatiquement le 30 juillet 2020.

\section{(c) (i) (2) $\Theta$}

Appareil est mis à disposition selon les termes de la Licence Creative Commons Attribution - Pas d'Utilisation Commerciale - Pas de Modification 4.0 International. 


\title{
Prise de site
}

\author{
Jean-Louis Déotte
}

\section{NOTE DE L'AUTEUR}

L'expression « prise de site » a été proposée par Jacques Boulet pour remplacer celle d'« in situ », trop vague et donnant lieu à trop d'expositions hétérogènes. Une première version de cet article a été publiée en juillet 2018, dans la rubrique Galerie d'Appareil. Il est consacré à la prise de site, par des œuvres contemporaines, d'un édifice patrimonial.

1 Il s'agit de distinguer ce qui a eu lieu et aura lieu dans l'Hôtel-Dieu de Tonnerre depuis les premières installations de Caroline Coppey, Didier Guth et Germain Roesz (2014-2015-2016) - de ce qui pourrait avoir lieu dans un espace comme la Pharmacie ${ }^{1}$, où les œuvres n'enchaînent pas sur l'espace. La quasi-totalité des lieux d'exposition sont, à l'instar de la Pharmacie, faits pour des œuvres ne requérant qu'une boîte blanche. Certes, cette dernière a une histoire très paradoxale, comme le rappellent René Vinçon ou Sandrine Morsillo, mais c'est une autre affaire.

2 Un lieu comme l'Hôtel-Dieu est une «singularité ontologique » (Henri Focillon). En l'occurrence, il s'agit d'un édifice patrimonial régi par des règlements. Or un espace d'exposition peut être quelconque, ce peut même être un non-lieu.

À dire vrai, l'expression «prise de site" suppose l'expérience des installations précitées, et si elle est devenue comme un programme artistique, c'est qu'elle s'est imposée par la pratique expérimentale au point de devenir impérative.

Il y a eu et il y aura dans l'Hôtel-Dieu des expositions indifférentes au lieu, à sa destination onto-théologique, à son histoire, à son architecture... mais elles n'entrent pas dans le champ de compétence de l'association Art et Patrimoine Hospitalier en Tonnerois (AHPT). Cette association promeut des expositions qui sont des réponses au défi lancé aux artistes par cet édifice exceptionnel.

5 La réponse de Caroline Coppey, ce fut l'introduction d'une sorte de grande verrière, comme un vitrail gothique (à l'image de ceux de Chartres), mais constituée de chiffons 
colorés, tous singuliers. D'Orient en Occident, selon le déplacement du soleil, mais aussi selon la genèse constructive de l'édifice, l'installation filtrait ou reflétait la lumière pour un spectateur se tenant sur la tribune de la nef. Tribune qui pourrait devenir le lieu privilégié d'observations esthétiques ultérieures. Cependant, à la différence d'un vitrail médiéval, l'installation ne narrait rien; elle ne dépendait pas du texte sacré. Séparé du lieu, le Grand Voile devenait une œuvre à part entière, livré au jeu herméneutique, ce qui n'est pas le cas des installations prises de site.

6 La réponse de Didier Guth, quant à elle, prit la forme d'un parcours eschatologique, scandé par le rythme des entraits, menant d'un couloir étroit - moment d'un véritable rite de passage (les ossements dans des sarcophages de pierre : la mort terrestre) - à la chapelle du Revestiaire: le Sépulcre, œuvre majeure de l'École bourguignonne (les frères Sonnette étaient deux élèves de Claus Sluter), la promesse de la Résurrection. À l'évidence, les formes blanches et perforées de cette échelle, partant du niveau de l'entrée pour aboutir à la chapelle du Revestiaire, n'étaient pas conçues comme des œuvres potentielles, autonomes, des suspens pouvant s'accomplir dans un musée.

7 Enfin, la réponse de Germain Roesz, à partir d'un pattern - un triangle, un arc de cercle - repris systématiquement sur différents draps de couleur diaphane descendant des poutres, reconstituait les enclos des lits des malades et la segmentation fonctionnelle probable de la nef. À y regarder de près, l'arc de cercle du pattern ne renvoie pas aux arcs de la voûte (plein cintre surbaissé), mais plutôt à un élément de voûte en chaînette, comme si l'artiste avait voulu rendre impossible l'identification politicoculturelle à l'arc roman, si présent dans l'imaginaire bourguignon, comme le sont les tuiles vernissées et colorées, disposées géométriquement à Beaune. Cet élément du pattern de l'artiste est une sorte de critique architecturale de la structure médiévale de l'édifice: une voûte en berceau ayant besoin de contreforts extérieurs pour se maintenir, alors que la voûte en chaînette se soutient elle-même: c'est un idéal constructif pour Simondon.

On le comprend, d'un édifice dont on ne sait trop comment il fut conçu dans les premiers temps du règne de Marguerite de Bourgogne, sinon qu'il répondait à une commande hospitalière au sens large, les artistes reconstituèrent les éléments d'une utopie gisant toujours dans le passé et à faire surgir du vide.

Du fait d'un processus historique qui aboutit à la patrimonialisation de la nef-halle (Jacques Boulet), et si l'on se réfère au plan élaboré par Camille Dormois en 1852-1853, il apparaît que la cité hospitalière extérieure ${ }^{2}$ a été quasiment détruite. À cela s'ajoute l'évidement du bâtiment lui-même qui a dû subir une transformation considérable quand il fut réalisé comme église en 1648. Espérons que les travaux menés sur les plans du XVII ${ }^{\mathrm{e}}$ siècle par Julie Jaupitre permettront d'avoir une idée plus précise des fonctions préhistoriques de la nef ${ }^{3}$.

10 On entendra ici le terme "préhistorique » à la manière de Walter Benjamin : ce qui est "préhistorique », c'est l'originaire, la structure d'origine. Aujourd'hui, les artistes font toutefois l'expérience d'un immense espace vide mis à disposition, donc sans destination. Ce que nous attendons d'eux, c'est de rendre visible le "préhistorique ». Non pas comme une utopie du passé, mais comme utopie nous attendant toujours, s'adressant à nous. La pratique artistique aura été un regard jeté vers un passé qui a toujours sa charge de sens. Le travail des artistes ne fut pas, en effet, strictement archéologique ; même s'il n'est pas ignorant, il est peut-être à prendre comme celui d'un Viollet-le-Duc livrant, par ses dessins, une reconstitution du lieu « tel qu'il fut en 
vérité " selon lui, mais en vérité fort romanesque. Car l'artiste, chez lui, domine le chartiste. Cette prétention de dire le vrai et de le faire voir est, au fond, de nature positiviste ; comme l'était celle de Fustel de Coulanges dans La Cité antique, car si une telle prétention pouvait se réaliser, elle invaliderait la charge utopique. Mais si l'on veut sauver cette reconstitution, car après tout, elle inspira son gendre Oradou pour la salle des malades des Hospices de Beaune, laquelle fit retour comme fantasmagorie pour l'Hôtel-Dieu au xxe siècle, il faut alors la déclarer artistique, elle aussi.

11 Les touristes qui visitent aujourd'hui Beaune, avec le succès que l'on sait, parcourent un rêve romantique réalisé et le prennent pour la "vérité historique ». Or cette vérité est datée, comme le sont toujours les traductions d'un texte: raison pour laquelle un traducteur chasse l'autre. Ces « interprétations » obéissent souvent aux exigences d'un sauvetage, comme à Vézelay, tout en visant une vérité « préhistorique ».

On comprend qu'à Tonnerre certains aient voulu reconstituer une salle des malades comme ce fut le cas à Beaune, mais s'ils s'inspiraient des dessins de Viollet-le-Duc, pourquoi ne voulurent-ils pas faire recouvrir l'ensemble de la toiture de tuiles vernissées (ce qu'indique Viollet-le-Duc) ? Ce qui avait conduit ce dernier à imaginer un dispositif (au sens foucaldien du terme) de lits cloisonnés, perpendiculaires aux murs, surplombés d'une galerie dite de surveillance et d'aération (ouverture des grandes baies géminées), ce sont les trous réguliers que l'on trouve positionnés en vis-à-vis dans les murs gouttereaux, en bas des baies.

Figure 1. L'Hôtel-Dieu de Tonnerre dans le Dictionnaire raisonné de l'architecture française $d u x l^{e} a u x v I^{e}$ siècle de Viollet-le-Duc

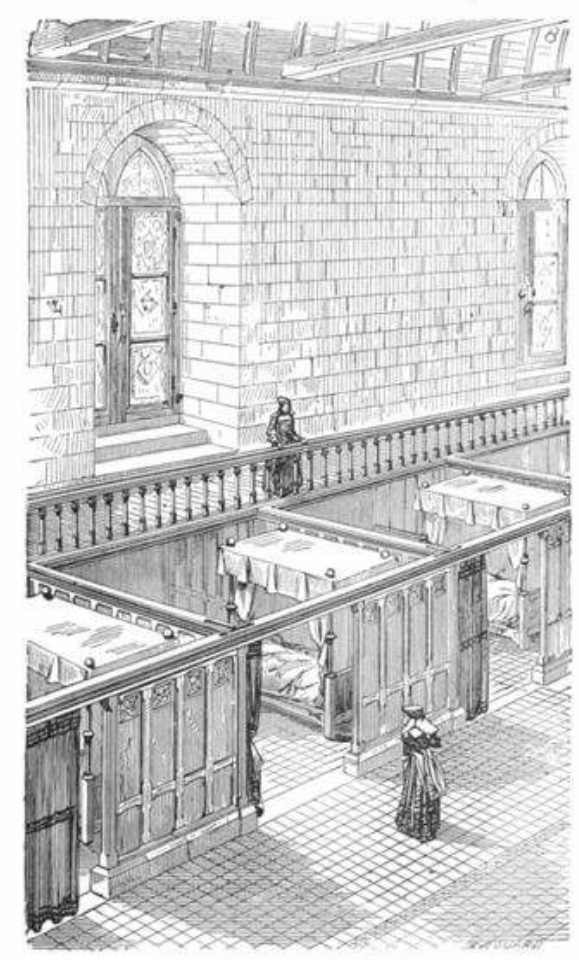

Source : Édition Bance-Morel (1854-1868)

S'ils servaient de points d'ancrage aux lits et donc à la galerie parcourant tout l'édifice - ce qui pose problème pour franchir la nef à la hauteur de l'actuel chœur -, leur 
fonction est largement sous-estimée, tandis qu'au-dessus des baies d'autres trous supportent les entraits. Que la disposition des lits soit parallèle aux murs ou perpendiculaire, il n'en reste pas moins qu'un vaste espace central demeurait non fonctionnel sous une hauteur considérable. En comparaison, la reconstitution de la salle des malades des Hospices de Beaune est beaucoup plus réaliste, avec de surcroît une circulation discrète à la périphérie de la salle et la possibilité, pour chaque malade, d'assister à la messe depuis son lit ! C'est le surdimensionnement de Tonnerre qui pose problème : trois fois Beaune en volume.

Il faut concevoir autrement le volume de Tonnerre: on peut parfaitement imaginer qu'une première série d'entraits supportait un plancher et que la halle, aujourd'hui totalement dégagée, comportait deux niveaux. Comment justifier cette hypothèse utopique?

14 Nous proposons de constituer la série des édifices à plan basilical à deux niveaux, dont le prototype pourrait être constitué par l'Échiquier du château de Caen (XII siècle) et par un magnifique exemplaire : la grande salle d'apparat du château de Montargis. Il s'agit, le plus souvent, de salles princières et/ou synodales (cf. château de Sens).

Figure 2. Représentation en coupe de la grande salle du château de Montargis (1576)

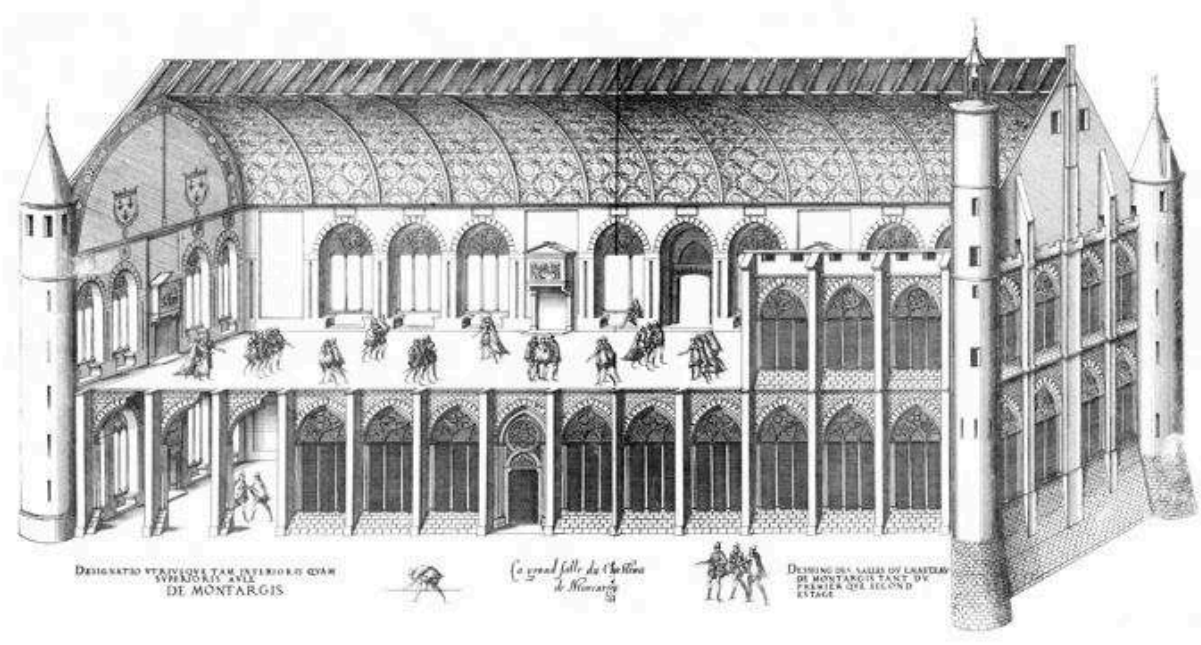

Gravure de Jacques Ier Androuet du Cerceau, Les dessins des plus excellents bastiments de France Source : Paris, Gilles Beyes, 1576-1579

On peut alors se demander comment Viollet-le-Duc a pu imaginer l'aménagement ciprésent de Tonnerre, alors que manifestement ni la galerie au-dessus des lits (dont la disposition est purement hypothétique), ni la structure des lits eux-mêmes n'avaient besoin d'une telle poutraison révélée par les trous dans les murs gouttereaux! D'autant qu'on ne voit pas la nécessité d'une nef aussi vaste pour une quarantaine de lits !

Au contraire, l'hypothèse d'un édifice à deux niveaux permet de réaliser l'intégralité du programme de Marguerite de Bourgogne. D'autant que si l'on considère que le sol a été surélevé $(1,50 \mathrm{~m}$ de remblais) pour transformer la salle en cimetière et pour lutter contre les infiltrations d'eau, dès lors le niveau de l'actuel cellier est le bon. D'autres 
indications vont dans ce sens : l'existence de fossés entourant l'édifice, celle de portes basses et de portes hautes, aujourd'hui inutiles.

On suggère que le premier niveau permettait d'accueillir les pèlerins, les étrangers de passage, le peuple des affamés et des pauvres, ainsi que les réserves, la cuisine, le cellier, les cellules des sœurs, etc.

L'étage noble, fort bien éclairé et aéré, aurait été celui des malades.

18 Avec des rayons laser, on peut dessiner dans l'espace, suggérer des volumes, mais aussi édifier des pans de mur colorés et parfaitement évanescents. Une architecture de fiction dans l'architecture patrimoniale! On pourra dire alors : voici ce que le passé aurait pu être !

D'autres éléments de l'édifice pourraient servir de ligne de mire. Le tombeau de Louvois, œuvre insigne de Girardon, ne cesse de provoquer celui de Marguerite de Bourgogne, la bienfaitrice, aux côtés duquel il trône. Il faudrait faire sortir de l'ombre ce ministre de la Guerre de Louis XIV qui fit dévaster villes et campagnes du Palatinat lors de la seconde campagne d'Allemagne, arrachant les vergers pour faire table rase, déportant les populations vers l'Alsace, etc. Celui-ci est probablement à l'origine de la haine durable que portèrent les Hollandais et les Allemands aux Français, et qui est encore évidente au château de Heidelberg, où la Tour rompue porte le nom de Tour des Français.

De même que Jochen Gerz a développé l'idée esthétique d'« anti-monument », parce qu'un monument est toujours une œuvre oublieuse, un moyen d'enterrer définitivement l'événement, il faudrait, s'agissant des grands massacreurs de l'humanité, parasiter leurs statues selon la proposition de Peter Gnass ${ }^{4}$, et rappeler les lieux où furent les hommes maléfiques. Nous manquons d'installations de déniaisement qui ne risquent pas, à leur tour, de devenir des monuments.

\section{NOTES}

1. Nouvel espace pour l'art contemporain situé dans le pavillon Dormois de l'hôpital de Tonnerre. À propos de la Pharmacie, voir notamment notre article «L'exposition "Prendre soin" comme déniaisement », Appareil [En ligne], Galerie, mis en ligne le 28 août 2018, consulté le 02 octobre 2018. URL : http://journals.openedition.org/appareil/2641 ; DOI : 10.4000/appareil.2641

2. À l'exception du bâtiment carré dit du Pressoir, le long du bief de l'Armançon.

3. Voir Le Clech-Charton Sylvie, L'hôtel-Dieu de Tonnerre. Métamorphose d'un patrimoine hospitalier, $\mathrm{XIII}$-XXe siècle, Langres, Éditions Dominique Guéniot, 2012. Cet ouvrage constitue notre indispensable référence. On y apprend «qu'en 1555 des travaux sont exécutés se rapportant à une galerie [intérieure] dont les structures de bois sont décrites comme encastrées dans des trous pratiqués dans les murs» (p. 46). Ce sont probablement ces documents qu'utilisera Viollet-le-Duc dans sa reconstitution : voir son Dictionnaire raisonné de l'architecture $d u \mathrm{XI}^{e} a u \mathrm{XV}^{e}$ siècle, édition Bance-Morel, 1854-1868. Mais quelle était la structure antérieure du bâtiment? N'y avait-il que des lits disposés en parallèle le long des murs, dans cette immense nef? 
4. Gnass Peter, La Multitude déchue. Exposition de la Maison de la culture de Côte-des-Neiges, Montréal, 13 octobre au 11 décembre 2016, Montréal, Éditions Périmètre, Concepts en art contemporain, 2016.

INDEX

Mots-clés : tradition, exposition, suspension, musée, esthétisation, nouveauté, analogie

\section{AUTEUR}

\section{JEAN-LOUIS DÉOTTE}

Jean-Louis Déotte a été professeur de philosophie à l'Université Paris VIII, Saint-Denis, coordinateur du thème « Esthétique, arts et industries » à la MSH Paris Nord, directeur de la collection « Esthétiques » chez L'Harmattan et enfin, directeur et fondateur de la revue Appareil. 